\title{
1 Die Medizinische Mikrobiologie im 21. Jahrhundert
}

\author{
S. Suerbaum, H. Hahn
}

\section{$>$ Einleitung}

Die Medizinische Mikrobiologie befasst sich mit der ursächlichen Rolle von pathogenen (d.h. krankheitserzeugenden) Mikroorganismen bei der Entstehung von Störungen im Funktionsablauf des menschlichen Organismus. Störungen dieser Art entstehen durch Ansiedlung und Vermehrung von Mikroorganismen im Sinne des Parasitismus; sie treten als Infektionskrankheit in Erscheinung. Demgemäß betrachtet man die parasitierenden Mikroorganismen als Krankheitserreger; das befallene Individuum wird als "Wirt« oder als "Makroorganismus« bezeichnet. Da bei der Betrachtung von Infektionen sowohl der Wirt mit seinen Reaktionen als auch die krankheitserzeugenden Eigenschaften eines Mikroorganismus (d. h. seine Pathogenität) im Vordergrund stehen, lässt sich die Medizinische Mikrobiologie am ehesten als Infektionslehre begreifen - als Lehre von der Auseinandersetzung des Wirts mit den krankheitserzeugenden Eigenschaften des Erregers.

\subsection{Gegenstand}

Das Gebiet der Infektionskrankheiten unter Einschluss der Immunologie bietet besonders klare und einprägsame Beispiele zur Darstellung von allgemeinen Prinzipien der Krankheitsentstehung. Die Krankheitserreger (•Tab. 1.1) stammen entweder aus der Umwelt oder aus der physiologischen Standortflora des betroffenen Individuums:

- Ein großer Teil der Krankheitserreger gehört zu den einzelligen Mikroorganismen; es sind entweder Bakterien, Pilze oder Protozoen.

- Ein anderer Teil wird zu den subzellulären Partikeln gerechnet; dies gilt für die Viren und die Prionen.

- Schließlich können auch vielzellige Organismen (Metazoen) als Krankheitserreger in Erscheinung treten; hierher gehören die parasitischen Würmer.
Bakterien sind Prokaryonten, Pilze bilden ein eigenes Reich innerhalb der Eukaryonten, während Protozoen und Metazoen zum Tierreich gehören.

Die in der Alltagssprache als Ungeziefer bezeichneten Arthropoden (Läuse, Wanzen, Zecken, Milben u. a.) spielen eine überaus wichtige Rolle als Überträger von Krankheitserregern. Die Kenntnis ihrer Biologie bildet vielfach die Grundlage einer wirksamen Bekämpfung von Infektionskrankheiten.

In den letzten Jahren ist zunehmend klar geworden, dass die Bakterien, die physiologisch große Bereiche der äußeren und inneren Körperoberflächen besiedeln, für die Funktion des Körpers und besonders des Immunsystems eine essenzielle Rolle spielen. Die Analyse der Interaktion von solchen Kommensalen mit dem Wirt gehört ebenfalls zum Bereich der Medizinischen Mikrobiologie.

\subsection{Aufgabenstellung}

Die Aufgabenstellung der Medizinischen Mikrobiologie wird von zwei Grundfragen bestimmt.

- Die eine bezieht sich auf die biologischen Besonderheiten der Krankheitserreger.

- Die andere betrifft diejenigen Vorgänge, welche im Wirtsorganismus ausgelöst und als Infektion bezeichnet werden; es sind dies Schädigungsprozesse und Abwehrreaktionen.

Die Schädigungsprozesse sind die direkte Ursache der Krankheit; in ihrer Gesamtheit werden sie als Pathogenese bezeichnet. Die Abwehrreaktionen können zur Milderung der Krankheit, zur Heilung und zur Immunität führen. Manchmal schädigen sie den Wirtsorganismus selbst; dann spricht man von Immunpathogenese.

Die Kenntnis der biologischen Besonderheiten der Krankheitserreger, von der Natur der Schädigung und vom Wesen der

\begin{tabular}{|c|c|c|c|c|c|}
\hline & Viren & Bakterien & Pilze & Protozoen & Würmer \\
\hline DNA + RNA & - (DNA oder RNA) & + & + & + & + \\
\hline Ribosomen & - & + & + & + & + \\
\hline Zellkern & - & - (Kernäquivalent) & + & + & + \\
\hline Größe & $0,02-0,3 \mu \mathrm{m}$ & $0,2-10 \mu \mathrm{m}$ & $>0,7 \mu \mathrm{m}$ & $5-50 \mu \mathrm{m}$ & $60 \mu \mathrm{m}->10 \mathrm{~m}$ \\
\hline ein-/mehrzellig (e/m) & - & e & $\mathrm{e} / \mathrm{m}$ & e & M \\
\hline
\end{tabular}


Abwehrvorgänge ist von großer Bedeutung für die Bekämpfung der Infektionskrankheiten. Die zu diesem Zweck eingeleiteten Maßnahmen beziehen sich zu einem großen Teil auf das erkrankte Individuum, zum anderen Teil auf die gesamte Bevölkerung und deren Lebensraum. Im Einzelnen unterscheidet man die im Folgenden aufgeführten Maßnahmen.

Erregerdiagnose. Das ist die exakte Bestimmung der Krankheitsursache, nämlich des Erregers. Diese umfasst die Maßnahmen bei Abnahme von Untersuchungsmaterial und dessen Transport ins Labor durch den behandelnden Arzt, die Anwendung der Labormethoden durch den medizinischen Mikrobiologen sowie die Interpretation des Befundberichts aus dem Labor - letztere eine gemeinsame Aufgabe des behandelnden Arztes und des Mikrobiologen.

Kausalbehandlung. Das ist die Behandlung des Kranken durch Bekämpfung der Krankheitsursache, des Erregers, mittels Antibiotika bzw. Antikörper oder Virustatika.

Prävention (Infektionsverhütung). Hierzu gehören:

- Die Verminderung der Erreger-Emission vom Infizierten durch dessen Isolierung und durch Desinfektion seiner Ausscheidungen.

- Die Verkleinerung des Erreger-Reservoirs, z. B. durch Rattenbekämpfung bei der Pest.

- Die Unterbrechung des Übertragungsvorgangs, durch die Überprüfung und Elimination von kontaminierten Lebensund Arzneimitteln oder die gezielte Vernichtung von übertragungsfähigen Arthropoden (Vektoren), z. B. bei der Schlafkrankheit.

- Prophylaktische Schutzimpfung, z. B. gegen Hepatitis B, Poliomyelitis, Diphtherie.

- Prophylaktische Gabe von Chemotherapeutika bei Exponierten, z. B. bei Malariagefahr.

Epidemiologie. Die epidemiologische Analyse liefert die Möglichkeit, Vorkommen und Ausbreitung von Infektionskrankheiten innerhalb eines größeren Gebiets zu analysieren und daraus Gesetzlichkeiten abzuleiten.

Die Medizinische Mikrobiologie ist Gegenstand einer eigenständigen Facharztweiterbildung. Nach 5 Jahren Weiterbildung (davon 1 Jahr in der Klinik) kann der Titel des Facharztes für Mikrobiologie, Virologie und Infektionsepidemiologie erworben werden.

\subsection{Heutige Bedeutung}

Mit der Einführung der antiinfektiven Chemotherapeutika, die nach dem 2. Weltkrieg zu breiter Anwendung gelangten, bildete sich in den 1960er Jahren die Überzeugung, dass Infektionskrankheiten in absehbarer Zeit der Vergangenheit angehören würden. Unterstützung fand diese Auffassung durch die erfolgreiche Ausrottung der Pocken, die am 8.5.1980 durch die Weltgesundheitsorganisation proklamiert wurde. Dieses Ereignis gab Anlass zu Voraussagen namhafter Infektionsforscher, dass mit der Ausrottung weiterer Seuchen und letztlich mit dem Ende der Infektionskrankheiten insgesamt zu rechnen sei.

Wie falsch diese Auffassung war, sollten uns die Mikroorganismen alsbald lehren (•Tab. 1.2): Infektionskrankheiten sind heute die häufigste Todesursache weltweit: 35\% aller Menschen sterben an Infektionen - und kein Ende in Sicht!

Die Ursachen für diese Entwicklungen sind vielfältig:

\subsubsection{Resistenzentwicklung}

Der massive und z. T. unsachgemäße Antibiotikaeinsatz hat durch den hierdurch ausgeübten Selektionsdruck zur Verbreitung zahlreicher Erregerstämme geführt, die hochresistent gegen die gebräuchlichen Antibiotika geworden sind. Beispiele hierfür sind:

- Methicillin-resistente Staphylokokken (MRSA)

- Vancomycin-resistente Enterokokken (VRE)

- Penicillin-resistente Gonokokken

- multiresistente Tuberkulose-Erreger (MDR und XDR)

- zahlreiche hochresistente gram-negative Stäbchenbakterien

\subsubsection{Auftreten neuer Krankheitserreger}

Die -Tab. 1.2 zeigt eindrücklich, wie viele bisher unbekannte Krankheitserreger in den vergangenen 30 Jahren aufgetreten sind. Zum Teil sind diese neu entstanden, wie z. B. die beiden humanen HI-Viren (HIV-1, HIV-2), die vermutlich aus bei Affen vorkommenden Immundefizienz-Viren (SIV) hervorgegangen sind und einen "Wirtssprung « vollzogen haben. Ökologische Veränderungen können für manche Erreger die Ausbreitungsbedingungen verbessern, sodass sie aus ihrem bisherigen abgeschiedenen Habitat auch in von Menschen besiedelte Gebiete gelangen. Oder aber altbekannte Krankheitserreger erscheinen in neuem Gewande und erzeugen auf vielfache Art und Weise bisher unbekannte Krankheitsbilder. Ein gutes Beispiel sind E. coliStämme, die durch Akquisition ihre Ausstattung mit Virulenzfaktoren dauernd ändern.

Weitere Beispiele sind: SARS-Viren (Pneumonie), Helicobacter pylori (B-Gastritis, Ulcus duodeni, Magenkrebs), Legionellen (Pneumonie) (-Tab. 1.2). Teilweise haben die durch sie hervorgerufenen Infektionskrankheiten schon jetzt verheerende Ausmaße angenommen und sind im Begriff, ganze Erdteile demografisch zu verändern, - so z. B. AIDS in Afrika südlich der Sahara. 
- Tab. 1.2. Seit 1972 identifizierte Erreger von Infektionskrankheiten

\begin{tabular}{|c|c|c|}
\hline Jahr & Erreger & Krankheit \\
\hline 1972 & $\begin{array}{l}\text { Small round structured viruses } \\
\text { (SRSVs; Caliciviren) }\end{array}$ & Diarrhoe (Ausbrüche) \\
\hline 1973 & Rotaviren & Diarrhoe (weltweit) \\
\hline 1975 & Astroviren & Diarrhoe (Ausbrüche) \\
\hline 1975 & Parvovirus B19 & Erythema infectiosum; aplastische Krise bei chronischer hämolytischer Anämie \\
\hline 1976 & Cryptosporidium parvum & Akute Enterocolitis \\
\hline 1977 & Ebolavirus & Ebola hämorrhagisches Fieber \\
\hline 1977 & Legionella pneumophila & Legionellose \\
\hline 1977 & Hantaan Virus & Hämorrhagisches Fieber mit renalem Syndrom \\
\hline 1977 & Campylobacter spp. & Diarrhoe \\
\hline 1980 & Humanes T-Zell-Leukämie Virus-1 (HTLV-1) & Adulte T-Zell-Leukämie/Lymphom; tropische spastische Paraparese \\
\hline 1982 & Humanes T-Zell-Leukämie Virus-2 (HTLV-2) & Atypische Haarzell-Leukämie (T-Zelltyp) \\
\hline 1982 & Borrelia burgdorferi & Lyme-Borreliose \\
\hline 1983 & Humane Immundefizienz-Viren (HIV-1, HIV-2) & Erworbenes Immundefizienzsyndrom (AIDS) \\
\hline 1983 & Escherichia coli 0157 (EHEC) & Diarrhoe; hämorrhagische Kolitis; hämolytisch urämisches Syndrom \\
\hline 1983 & Helicobacter pylori & $\begin{array}{l}\text { Gastritis; Magen- und Duodenalgeschwüre; erhöhtes Magenkarzinomrisiko, } \\
\text { MALT-Lymphom des Magens }\end{array}$ \\
\hline 1988 & Humanes Herpesvirus-6 & Exanthema subitum (Roseola infantum; Drei-Tage-Fieber) \\
\hline 1989 & Ehrlichia spp. & Humane Ehrlichiose \\
\hline 1989 & Hepatitis C-Virus (HCV) & Hepatitis C \\
\hline 1989 & Guanarito-Virus & Venezolanisches hämorrhagisches Fieber \\
\hline 1990 & Humanes Herpesvirus-7 & Exanthema subitum; Pityriasis rosea \\
\hline 1990 & Hepatitis E-Virus (HEV) & Hepatitis E \\
\hline 1992 & Vibrio cholerae 0139 & neue Variante assoziiert mit epidemischer Cholera \\
\hline 1992 & Bartonella henselae & Katzenkratzkrankheit; kutane Angiomatose \\
\hline 1993 & Sin Nombre-Virus & Hantavirus Lungensyndrom (»Four corners disease«) \\
\hline 1993 & Hepatitis G-Virus (HGV) & niedriger Krankheitswert \\
\hline 1994 & Sabia Virus & Brasilianisches hämorrhagisches Fieber \\
\hline 1994 & Humanes Herpesvirus-8 (HHV-8) & $\begin{array}{l}\text { Kaposi-Sarkom; primäres Lymphom der Körperhöhlen; } \\
\text { Castleman-Krankheit }\end{array}$ \\
\hline 1994 & Hendravirus, equines Morbillivirus (EMV) & Meningitis; Enzephalitis \\
\hline 1996 & Prionprotein & Transmissible spongiforme Enzephalopathien (TSE) \\
\hline 1997 & Influenza A-Virus (H5N1) & Influenza (Hongkong) \\
\hline 1997 & Transfusion-transmitted virus (TTV) & möglicherweise Hepatitis \\
\hline 1998 & Nipahvirus & Meningitis; Enzephalitis \\
\hline 1999 & Influenza A-Virus (H5N9) & Influenza (Hongkong) \\
\hline 2003 & SARS associated Coronavirus & Schweres Akutes Respiratorisches Syndrom (SARS) \\
\hline 2004 & Hochpathogene aviäre Influenza-Viren (H5N1) & Erstmaliger Nachweis als Infektionserreger beim Menschen \\
\hline
\end{tabular}




\subsubsection{Soziale Faktoren, wie Armut, Zuwanderung, Urbanisierung}

Diese Faktoren leisten zusammen mit AIDS v. a. der Ausbreitung der Tuberkulose Vorschub (»AIDS und Tb: double trouble $)$.

\subsubsection{Massentourismus}

»Wer viel herumkommt, fängt sich viel ein«. Gerade die sexuell übertragenen Erkrankungen und die Nahrungsmittelinfektionen profitieren von der großen Beweglichkeit des modernen Menschen, indem sie von Erdteil zu Erdteil verschleppt werden.

Auf diese Weise sorgen die Mikroorganismen dafür, dass wir permanent auf der Hut und weiterhin gegenüber überraschenden Attacken gewappnet sein müssen; sie sorgen auch dafür, dass mikrobiologischer/infektiologischer Sachverstand gefragt sein wird - bis weit in die Zukunft!

\section{In Kürze}

Die Medizinische Mikrobiologie befasst sich mit den krankmachenden Eigenschaften der Erreger und den Reaktionsformen des befallenen Makroorganismus (Infektionen). Sie stellt Methoden zur Diagnose von Krankheitserregern zur Verfügung und trägt zur Therapie bei, indem sie die Empfindlichkeit der Krankheitserreger gegen Chemotherapeutika prüft. Die Entwicklung und Anwendung von Methoden zur Prävention von Infektionskrankheiten sind ebenfalls Aufgabe des Mikrobio- logen. Insoweit ist die Medizinische Mikrobiologie ein Teilgebiet der Infektionsmedizin. Neue Erreger, veränderte Erreger und veränderte gesellschaftliche Verhaltensweisen begünstigen die Entwicklung von Infektionskrankheiten und ihre Ausbreitung. Noch immer stirbt 1/3 aller Menschen an Infektionen und dies trotz erheblicher Fortschritte in der Kenntnis der Erreger und der ErregerWirtsbeziehung und der Entwicklung neuer Heilmethoden und Medikamente, und ein Ende der Entwicklung ist nicht in Sicht. 